\title{
Video Article \\ Glycoproteomics of the Extracellular Matrix: A Method for Intact Glycopeptide Analysis Using Mass Spectrometry
}

\author{
Javier Barallobre-Barreiro ${ }^{1}$, Ferheen Baig ${ }^{1}$, Marika Fava ${ }^{1}$, Xiaoke Yin ${ }^{1}$, Manuel Mayr ${ }^{1}$ \\ ${ }^{1}$ King's British Heart Foundation Centre, King's College London
}

Correspondence to: Manuel Mayr at manuel.mayr@kcl.ac.uk

URL: https://www.jove.com/video/55674

DOI: doi:10.3791/55674

Keywords: Biochemistry, Issue 122, Extracellular matrix, fibrosis, glycoprotein, systems biology, proteomics, cardiovascular

Date Published: $4 / 21 / 2017$

Citation: Barallobre-Barreiro, J., Baig, F., Fava, M., Yin, X., Mayr, M. Glycoproteomics of the Extracellular Matrix: A Method for Intact Glycopeptide Analysis Using Mass Spectrometry. J. Vis. Exp. (122), e55674, doi:10.3791/55674 (2017).

\section{Abstract}

Fibrosis is a hallmark of many cardiovascular diseases and is associated with the exacerbated secretion and deposition of the extracellular matrix (ECM). Using proteomics, we have previously identified more than $150 \mathrm{ECM}$ and ECM-associated proteins in cardiovascular tissues. Notably, many ECM proteins are glycosylated. This post-translational modification affects protein folding, solubility, binding, and degradation. We have developed a sequential extraction and enrichment method for ECM proteins that is compatible with the subsequent liquid chromatography tandem mass spectrometry (LC-MS/MS) analysis of intact glycopeptides. The strategy is based on sequential incubations with NaCl, SDS for tissue decellularization, and guanidine hydrochloride for the solubilization of ECM proteins. Recent advances in LC-MS/MS include fragmentation methods, such as combinations of higher-energy collision dissociation (HCD) and electron transfer dissociation (ETD), which allow for the direct compositional analysis of glycopeptides of ECM proteins. In the present paper, we describe a method to prepare the ECM from tissue samples. The method not only allows for protein profiling but also the assessment and characterization of glycosylation by MS analysis.

\section{Video Link}

The video component of this article can be found at https://www.jove.com/video/55674/

\section{Introduction}

Fibrosis is a hallmark of many diseases. Fibroblasts proliferate and differentiate towards highly synthetic phenotypes, which are associated with the exacerbated secretion and deposition of extracellular matrix $(\mathrm{ECM})^{1}$. Excessive ECM deposition can continue, even after the initial injury has abated, leading to functional impairment. Using proteomics, we have previously identified more than $150 \mathrm{ECM}$ and ECM-associated proteins in cardiac tissue $^{2,3}$. They are not only structural proteins, but also matricellular proteins and proteases that contribute to the continuous remodeling and dynamic adaptation of the heart. Notably, many ECM proteins are glycosylated ${ }^{4}$. This post-translational modification (PTM) involves the addition of sugar residues to certain amino acid positions, and it affects protein folding, solubility, binding, and degradation ${ }^{5}$.

There are two main glycosylation types that occur in mammals. (1) $\mathrm{N}$-glycosylation occurs at the carboxamido nitrogen of asparagine residues (Asn) within the consensus sequence Asn-Xaa-Thr/Ser, where Xaa is any amino acid except for proline. (2) In O-glycosylation, sugar residues attach to serine and threonine residues (Ser, Thr) or, to a much lesser extent, to hydroxyproline and hydroxylysine. While O-glycosylation can occur in a variety of protein groups, $\mathrm{N}$-glycosylation is restricted to secreted proteins or extracellular domains of membrane proteins ${ }^{5}$. This makes $\mathrm{N}$-glycosylation an attractive target when studying the ECM.

Proteomics sets a new standard for the analysis of protein changes in disease. Thus far, most proteomics studies have been focused on intracellular proteins ${ }^{6}$. This is mainly due to the following reasons. First, abundant intracellular proteins hamper the identification of scarce ECM components. This is particularly crucial in cardiac tissue, in which mitochondrial and myofilament proteins account for a large proportion of the protein content ${ }^{7}$. Second, integral ECM proteins are heavily cross-linked and difficult to solubilize. Lastly, the presence of abundant PTMs (i.e., glycosylation) alters the molecular mass, charge, and electrophoretic properties of peptides, affecting both the separation and the identification by liquid chromatography tandem mass spectrometry (LC-MS/MS). Over recent years, we have developed and improved a sequential extraction and enrichment method for ECM proteins that is compatible with subsequent mass spectrometry (MS) analysis. The strategy is based on sequential incubations.

The first step is performed with $\mathrm{NaCl}$, an ionic buffer that facilitates the extraction of ECM-associated and loosely bound ECM proteins, as well as newly synthesized ECM proteins. It is detergent free, non-denaturing, non-disruptive of cell membranes, and amenable for further biochemical assays ${ }^{8}$. Then, decellularization is achieved with sodium dodecyl sulfate (SDS). At this step, a low SDS concentration ensures membrane destabilization and the release of intracellular proteins whilst preventing the disruption of the more soluble non-integral ECM components. Finally, ECM proteins are extracted with a guanidine hydrochloride buffer $(\mathrm{GuHCl})$. GuHCl is effective in extracting heavily crosslinked proteins and proteoglycans from tissues such as tendons ${ }^{9}$, cartilage ${ }^{10}$, vessels ${ }^{11,12,13}$ and the heart ${ }^{2,3}$. We applied this biochemical fractionation, in combination with LC-MS/MS, to explore ECM remodeling in cardiovascular disease ${ }^{2,3,11,12,13,14}$. Recent advances in MS include 
novel fragmentation methods, such as combinations of higher-energy collision dissociation (HCD) and electron transfer dissociation (ETD), which allow for the direct analysis of intact glycopeptides ${ }^{3,15}$.

Here we describe a methodology to prepare ECM for MS analysis that allows for the analysis of protein composition, the identification of glycosylation sites, and the characterization of glycan forms. Compared to previous analyses of ECM glycosylation ${ }^{16}$, this methodology allows for the direct assessment of compositional changes in glycosylation profiles in a site-specific manner using MS. We have applied this methodology to cardiovascular tissues. However, it can also be applied, with minor modifications, to the study of the ECM in other tissue specimens and can provide unprecedented insights into ECM biology.

\section{Protocol}

The study was approved by the Wandsworth Local Research Ethics Committee (reference number: 06/Q0803/37) and received institutional approval from the research and development office. All patients gave written informed consent.

\section{Extraction of Extracellular Matrix Proteins}

NOTE: The human atrial tissues used for these experiments were obtained from the atrial appendages during cardiopulmonary bypass, just after the cardioplegic arrest of the heart. All samples were collected at St George's Hospital, London, UK. All tissue samples must be frozen at $-80{ }^{\circ} \mathrm{C}$. Do not use samples preserved with fixatives, such as paraformaldehyde, that cross-link proteins.

1. Prepare all extraction buffers in advance of experiments, as per Table 1, in order to minimize the time between the extraction steps. Perform all incubations at room temperature (RT) in a temperature-controlled environment (i.e., $\sim 20^{\circ} \mathrm{C}$ ) to ensure consistency between extractions.

2. Weigh $20-50 \mathrm{mg}$ of tissue. If several samples are to be extracted, cut and weigh them one by one to avoid the complete thawing of the tissues. Using a scalpel, dice the tissue into 3-4 smaller pieces (i.e., 2-3 mm) and place them together into $1.5 \mathrm{~mL}$ tubes.

3. Add $500 \mu \mathrm{L}$ of ice-cold phosphate-buffered saline (PBS; see Table 1 and the Table of Materials) and perform five washes to minimize blood contamination.

4. Extraction Step 1: Incubation with $\mathrm{NaCl}$ Buffer

1. After washing with PBS, place the samples into $1.5 \mathrm{~mL}$ tubes with screw caps. Add $\mathrm{NaCl}$ buffer (see Table 1) at 10 times (v:w) the tissue weight. Vortex the tubes at RT for $1 \mathrm{~h}$ at minimum speed (i.e., $600 \mathrm{rpm}$ ).

NOTE: A low vortex speed is critical to avoid the mechanical disruption of the tissue during this step. Use a foam adaptor to place all tubes during the extraction.

2. Transfer the extracts to new tubes and centrifuge at $16,000 \times \mathrm{g}$ for $10 \mathrm{~min}$ at $4{ }^{\circ} \mathrm{C}$. Store the extracts at $-20^{\circ} \mathrm{C}$ until use. Briefly wash the remaining tissue pellets with fresh $\mathrm{NaCl}$ buffer. Use the same type of buffer (i.e., $100 \mu \mathrm{L}$ of $\mathrm{NaCl}$ buffer) for washing to prevent the extraction of other proteins with different solubilities (i.e., proteins not extractable with $\mathrm{NaCl}$ ).

3. After washing, ensure the complete removal of the buffer to minimize overlap in protein content with subsequent extraction steps. Discard the $\mathrm{NaCl}$ buffer used for washing.

NOTE: The ratio between the buffer volume and tissue weight is important for a reproducible extraction. A 10:1 ratio (v:w) for the $\mathrm{NaCl}$ and SDS extractions and 5:1 for the $\mathrm{GuHCl}$ step provide sufficient amounts of protein without saturating the buffer. Protein concentrations are approximately $1-2 \mu \mathrm{g} / \mu \mathrm{L}$ after extraction.

\section{Extraction Step 2: Decellularization with SDS Buffer}

1. Add SDS buffer (Table 1) at ten times ( $\mathrm{v}: \mathrm{w}$ ) the tissue weight; the use of low SDS concentrations (i.e., $0.1 \%$ ) is critical to avoid the loss of ECM proteins during decellularization. Vortex the tubes at RT for $16 \mathrm{~h}$ at minimum speed (i.e., $600 \mathrm{rpm}$ ). NOTE: A low vortex speed minimizes mechanical disruption of the ECM.

2. Transfer the extracts to new tubes. Centrifuge at $16,000 \mathrm{xg}$ for $10 \mathrm{~min}$ at $4{ }^{\circ} \mathrm{C}$; store at $-20^{\circ} \mathrm{C}$ until use. Briefly wash the remaining tissue pellets with ${ }_{d d} \mathrm{H}_{2} \mathrm{O}$ to remove the SDS. Ensure the complete removal of the liquid after washing.

\section{Extraction Step 3: Incubation with GuHCI Buffer}

1. Add GuHCl buffer (Table 1) at five times (v:w) the tissue weight. Vortex the tubes at RT for $72 \mathrm{~h}$ at maximum speed (i.e., 3,200 rpm); vigorous vortexing facilitates the mechanical disruption of the ECM.

2. Transfer the extracts to new tubes. Centrifuge at $16,000 \times \mathrm{g}$ for $10 \mathrm{~min}$ at $4{ }^{\circ} \mathrm{C}$ and store at $-20{ }^{\circ} \mathrm{C}$ until use.

\section{Protein Quantification and Precipitation}

NOTE: Due to the presence of detergents, the SDS buffer is incompatible with direct protein quantification based on measurements of absorbance at $280 \mathrm{~nm}$. To ensure reproducible quantification, use colorimetric assays for all protein extracts ${ }^{17}$.

1. Quantification.

1. Prepare standards for a calibration curve using bovine serum albumin (BSA) serially diluted in the appropriate extraction buffer (i.e., $\mathrm{NaCl}$, SDS, or $\mathrm{GuHCl})^{17}$. During this time, thaw the sample extracts.

2. Dilute the samples in extraction buffer to obtain concentrations within the linear range of absorbance; a 1:10 dilution (v:v) yields satisfactory results. Dilute the $\mathrm{GuHCl}$ samples with an equal amount of $\mathrm{ddH}_{2} \mathrm{O}$ for quantification, as the colorimetric assay is not compatible with concentrations of $>4 \mathrm{M} \mathrm{GuHCl}$

3. Use a bicinchoninic acid (BCA)-based colorimetric assay ${ }^{17}$ (see the Table of Materials), following the manufacturer's instructions for assays in 96-well plates; it is recommended to perform at least duplicate measurements.

4. After incubating for $30 \mathrm{~min}$, take absorbance readings at a wavelength of $570 \mathrm{~nm}$ in order to calculate the protein concentration using the BSA standard calibration curve ${ }^{17}$. 
2. Protein Precipitation

1. Thaw $\mathrm{GuHCl}$ extracts at RT. Aliquot $10 \mu \mathrm{g}$ of protein for each sample into new tubes. For a direct glycopeptide analysis, aliquot $50 \mu \mathrm{g}$ Add 10 times the volume of ethanol and incubate overnight at $-20^{\circ} \mathrm{C}$.

NOTE: $\mathrm{GuHCl}$ is not compatible with further enzymatic reactions and most electrophoretic applications. The removal of $\mathrm{GuHCl}$ is required before deglycosylation and trypsin digestion. The solubility of $\mathrm{GuHCl}$ in ethanol and, conversely, the low solubility of proteins allow for the effective removal of $\mathrm{GuHCl}$ while yielding approximately a $98 \%$ recovery of proteins ${ }^{18}$.

2. Centrifuge the samples at $16,000 \times \mathrm{g}$ for $30 \mathrm{~min}$ at $4{ }^{\circ} \mathrm{C}$ and aspirate the supernatant. Take care not to disturb the precipitated pellet. Dry the pellets for $15 \mathrm{~min}$ using a vacuum concentrator (see the Table of Materials) at RT. NOTE: The protocol can be stopped here and the dried pellets stored at $-20^{\circ} \mathrm{C}$ until use.

3. Optionally, run a gel electrophoresis as quality control (QC, see the Supplemental Methods).

\section{Sequential Deglycosylation for the Assessment of N-glycosylation Site Occupancy}

1. During sample drying (see step 2.2.2), prepare the deglycosylation buffer containing debranching deglycosylation enzymes, as per Table 1 . See Table of Materials for product details.

2. Add $10 \mu \mathrm{L}$ of deglycosylation buffer containing enzymes to each sample. Ensure the appropriate pellet resuspension by performing a quick vortex and spin-down of samples.

NOTE: The removal of sugar monomers using debranching enzymes is essential for the subsequent and complete removal of O-linked complex saccharides and facilitates the later cleavage of N-linked sugars by PNGase-F.

3. Incubate for $2 \mathrm{~h}$ at $25^{\circ} \mathrm{C}$ to allow for the removal of heparan sulfate by heparinase II. Increase the temperature to $37^{\circ} \mathrm{C}$ and incubate for $36 \mathrm{~h}$ with gentle agitation.

NOTE: Given the low reaction volumes and prolonged incubation times, use incubator shakers and tightly pack multiple $1.5 \mathrm{~mL}$ tubes inside a $50-\mathrm{mL}$ conical tube leaning inside the incubator at approximately $45^{\circ}$.

4. After $36 \mathrm{~h}$, centrifuge the samples for $1 \mathrm{~min}$ at $16,000 \times \mathrm{g}$ and evaporate the $\mathrm{H}_{2} \mathrm{O}$ from the samples by using a vacuum concentrator at $\mathrm{RT}$ for approximately $45 \mathrm{~min}$.

5. Resuspend the dried samples with $10 \mu \mathrm{L}$ of $\mathrm{H}_{2}{ }^{18} \mathrm{O}$ containing $50 \mathrm{U} / \mathrm{mL}$ PNGase-F, which cleaves all asparagine-linked glycans in a deamidation reaction.

NOTE: The resulting aspartic acid carries an excess mass of $2.98 \mathrm{Da}$, indicative of the presence of $\mathrm{N}$-glycosylation during MS analysis.

6. Incubate for $36 \mathrm{~h}$ at $37^{\circ} \mathrm{C}$ under constant agitation in the incubator shaker.

\section{In-solution Trypsin Digestion}

NOTE: This step should be carried out for both non-deglycosylated (i.e., used for direct glycopeptide analysis) and deglycosylated samples (i.e., used for the assessment of glycan occupancy).

1. Use $10 \mu \mathrm{g}$ of total protein for the assessment of $\mathrm{N}$-glycosylation site occupancy (as indicated in the previous step). For samples intended for direct glycopeptide analysis, use $50 \mu \mathrm{g}$ of protein as the starting amount.

NOTE: The following steps are described for $10 \mu \mathrm{g}$ of protein. Scale up th evolumes (i.e., 5 times for $50 \mu \mathrm{g}$ ) as required.

2. Denature the proteins on each sample aliquot using $9 \mathrm{M}$ urea and $3 \mathrm{M}$ thiourea, with final concentrations of $6 \mathrm{M}$ urea and $2 \mathrm{M}$ thiourea, respectively (e.g., for a $10 \mu \mathrm{L}$ sample, $20 \mu \mathrm{L}$ of urea/thiourea).

3. Reduce the proteins by adding $100 \mathrm{mM}$ dithiotreitol (DTT, $3.33 \mu \mathrm{L}$, final concentration: $10 \mathrm{mM}$ ). Incubate at $37^{\circ} \mathrm{C}$ for $1 \mathrm{~h}$ with agitation at 240 rpm.

4. Cool down the samples to RT before performing the alkylation by adding $0.5 \mathrm{M}$ iodoacetamide ( $3.7 \mu \mathrm{L}$, final concentration: $50 \mathrm{mM})$. Incubate in the dark for $1 \mathrm{~h}$.

5. Use pre-chilled $\left(-20^{\circ} \mathrm{C}\right)$ acetone (6 times the volume of the sample) to incubate the samples overnight at $-20^{\circ} \mathrm{C}$. Precipitate by centrifugation at $14,000 \times \mathrm{g}$ for $25 \mathrm{~min}$ at $4{ }^{\circ} \mathrm{C}$.

6. Aspirate the supernatant. Take care not to disturb the precipitated pellet. Dry the protein pellets using a vacuum concentrator for 30 min at RT.

7. Resuspend in $20 \mu \mathrm{L}$ of $0.1 \mathrm{M}$ triethylammonium bicarbonate (TEAB) buffer, $\mathrm{pH} 8.2$, containing trypsin $(0.01 \mu \mathrm{g} / \mu \mathrm{L})$ and digest overnight at 37 ${ }^{\circ} \mathrm{C}$ and $240 \mathrm{rpm}$.

8. Stop the digestion by acidifying the samples with $10 \%$ trifluoroacetic acid (TFA, $2 \mu \mathrm{L}$ for a final concentration of $1 \%$ TFA).

\section{Peptide Cleanup Using C18 Columns}

NOTE: The removal of interfering contaminants from the peptide mixture after digestion reduces ion suppression and improves signal-to-noise ratios and sequence coverage. This step should be carried out for both non-deglycosylated and deglycosylated samples.

1. Activate the resin on the $\mathrm{C} 18$ spin plate (see the Table of Materials) using $200 \mu \mathrm{L}$ of methanol per well and centrifuge at $1,000 \times \mathrm{g}$ for $1 \mathrm{~min}$.

2. Wash by adding $200 \mu \mathrm{L}$ per well of $80 \%$ acetonitrile (ACN) and $0.1 \%$ TFA in $\mathrm{H}_{2} \mathrm{O}$. Centrifuge at $1,000 \times \mathrm{g}$ for $1 \mathrm{~min}$.

3. Equilibrate by adding $200 \mu \mathrm{L}$ per well of $1 \% \mathrm{ACN}$ and $0.1 \%$ TFA in $\mathrm{H}_{2} \mathrm{O}$. Centrifuge at $1,000 \times \mathrm{g}$ for 1 min. Repeat this step two more times.

4. Load the samples (the entire volume) from step 4 into the wells containing the resin and centrifuge at 1,500 x g for $1 \mathrm{~min}$. Reload the flowthrough a second time and repeat the centrifugation.

5. Wash by adding $200 \mu \mathrm{L}$ per well of $1 \% \mathrm{ACN}$ and $0.1 \%$ TFA in $\mathrm{H}_{2} \mathrm{O}$. Centrifuge at $1,500 \times \mathrm{g}$ for 1 min. Repeat this step two more times.

6. Elute the samples with $170 \mu \mathrm{L}$ per well of $50 \% \mathrm{ACN}, 0.1 \%$ TFA in $\mathrm{H}_{2} \mathrm{O}$. Centrifuge at $1,500 \times \mathrm{g}$ for 1 min. Repeat the previous step and combine the collected eluate.

7. Dry the eluate using a vacuum concentrator for $2 \mathrm{~h}$ at RT. If it is not being used immediately, keep the dried samples at $-80{ }^{\circ} \mathrm{C}$ until use. 
NOTE: Deglycosylated samples intended for protein identification only are ready to use for LC-MS/MS after this step. Steps 6 and 7 are not required for these samples.

8. Thaw and resuspend the deglycosylated samples in $2 \% \mathrm{ACN}$ and $0.05 \%$ TFA in $\mathrm{H}_{2} \mathrm{O}$ to a final protein concentration of $0.5 \mu \mathrm{g} / \mu \mathrm{L}$. Proceed with step 6 for the direct glycopeptide analysis of non-deglycosylated samples.

9. Optionally, filter the peptides prior to labeling with tandem mass tags (TMT); see the Supplemental Methods for peptide fitration.

\section{Labeling with TMT (for Direct Glycopeptide Analysis Only)}

1. Thaw and resuspend the dried pellets in $50 \mu \mathrm{L}$ of $50 \mathrm{mM}$ TEAB to obtain a concentration of $1 \mu \mathrm{g} / \mu \mathrm{L}$.

2. Resusped the $0.8-\mathrm{mg}$ vial of TMT Zero ( $\mathrm{TMT}^{0}$, see the Table of Materials) reagent in $41 \mu \mathrm{L}$ of ACN. Follow the manufacturer's recommendantions for resuspension.

3. Label the peptide samples at a ratio of $50 \mu \mathrm{g}$ peptides to $0.4 \mathrm{mg} \mathrm{TMT}^{0}$ (i.e., $50 \mu \mathrm{L}$ of peptides to $20.5 \mu \mathrm{L}$ of $\mathrm{TMT}^{0}$ ). Incubate at RT for $1 \mathrm{~h}$.

4. Quench the labeling reaction by adding $5 \%$ hydroxylamine at a ratio 6:100 (i.e., $4.23 \mu \mathrm{L}$ of $5 \%$ hydroxylamine). Incubate at RT for 15 min.

5. Dry the TMT ${ }^{0}$-labelled peptide samples for $1 \mathrm{~h}$ at RT using a vacuum concentrator. Resuspend in $10 \mu \mathrm{L}$ of ddH $\mathrm{d}_{2} \mathrm{O}$.

NOTE: Due to the glycan residues, glycopeptides display a higher molecular mass than non-glycosylated peptides. TMT $^{0}$ increases the charge state of glycopeptides. This reduces their relative mass to charge $(\mathrm{m} / \mathrm{z})$ ratios and facilitates ETD fragmentation.

\section{Glycopeptide Enrichment}

1. Use reaction buffers provided in the kit (see the Table of Materials).

2. Add $50 \mu \mathrm{L}$ of binding buffer to each $10 \mu \mathrm{L}$ sample from step 6.5. Vortex the glycocapture resin solution until it becomes homogeneous. Use a zwitterionic hydrophilic interaction liquid chromatography (ZIC-HILIC)-based capture ${ }^{15}$.

3. Aliquot $50 \mu \mathrm{L}$ of the resin suspension to new $1.5 \mathrm{~mL}$ tubes. Spin for $1 \mathrm{~min}$ at $2,500 \mathrm{xg}$ and remove the supernatant. Add $60 \mu \mathrm{L}$ of sample (i.e., the sample with binding buffer) to the tubes containing the resin pellets. Mix using a pipette and incubate at RT for 20 min in agitation at $1,200 \mathrm{rpm}$.

4. Centrifuge for $2 \mathrm{~min}$ at $2,000 \times \mathrm{g}$ and transfer the supernatant to new tubes. Keep the tubes. Add $150 \mu \mathrm{L}$ of wash buffer to the resin tubes. Mix using a pipette and incubate at RT for $10 \mathrm{~min}$ in agitation at 1,200 rpm.

5. Spin for $2 \mathrm{~min}$ at 2,500 $\mathrm{xg}$. Transfer the supernatant to the same tubes (from step 7.4). Repeat the washing steps two times.

6. Add $75 \mu \mathrm{L}$ of elution buffer and mix using a pipette. Agitate at $1,200 \mathrm{rpm}$ for $5 \mathrm{~min}$ at RT and then centrifuge the tubes for $2 \mathrm{~min}$ at $2,500 \mathrm{xg}$. Transfer the supernatants to new $1.5 \mathrm{~mL}$ tubes. Repeat the washing steps and then transfer the eluate supernatant to the same tube.

7. Centrifuge the tubes containing the eluate (i.e., glycopeptides) for $2 \mathrm{~min}$ at 2,500 $\mathrm{x}$ g. Transfer the supernatant to new tubes to ensure the removal of any remaining resin from the previous steps.

8. Dry the total $150 \mu \mathrm{L}$ of eluate using a vacuum concentrator for approximately $2 \mathrm{~h}$ at $\mathrm{RT}$. Resuspend the dried-down glycopeptides in $15 \mu \mathrm{L}$ of $2 \% \mathrm{ACN}$ and $0.05 \%$ TFA in $\mathrm{ddH}_{2} \mathrm{O}$.

9. Proceed to perform LC-MS/MS using HCD fragmentation to analyse the ECM protein composition, and LC-MS/MS using HCD and ETD fragmentation for glycopeptide characterization. See section 8.

\section{Mass Spectrometry Analysis}

1. Perform LC-MS/MS using HCD fragmentation to analyse the ECM protein composition; see the Supplemental Methods for details.

2. Perform LC-MS/MS using HCD and ETD fragmentation for glycopeptide characterization (see the Supplemental Methods for details); the enriched sample should be compared to the non-enriched input material ${ }^{15}$.

NOTE: Detailed descriptions of LC-MS/MS methods for indirect glycopeptide analysis, direct glycopeptide analysis, and database search are provided in the Supplemental Methods. Researchers interested in characterizing ECM protein and glycan composition using mass spectrometry are encouraged to refer to previous publications ${ }^{3,11,15}$.

\section{Representative Results}

A schematic workflow of the protocol is provided in Figure 1.

\section{ECM extraction protocol}

The efficiency of the extraction can be monitored by running aliquots form each extract on Bis-Tris acrylamide gels and using silver staining for visualization. Figure 2A shows the complementarity of the $\mathrm{NaCl}$, SDS and $\mathrm{GuHCl}$ extracts after sequential extraction. This QC allows for identification of potential issues with sample quality such as excessive protein degradation. After extraction, ECM glycoproteins are abundant in the $\mathrm{GuHCl}$ extracts (Figure 2B).

\section{Deglycosylation}

To assess the efficiency of deglycosylation, a non-deglycosylated control should be run in parallel. Deglycosylation times have to be suitable to achieve a complete and homogeneous removal of sugar residues, as exemplified in Figure 3A. Figure 3B shows a representative example of samples efficiently deglycosylated by the addition of enzymes for GAG removal and deglycosylation enzymes that target smaller $\mathrm{N}$ - and O-linked oligosaccharides. 


\section{Glycoproteomics}

The protocol for assessment of the occupancy of NxT/S sequons improves protein sequence coverage for ECM glycoproteins after MS (Figure 3C) and allows for an initial screening of the presence of glycoproteins. This helps to reduce the search time for glycopeptides, as databases can be customized to contain previously identified glycoproteins. HCD-ETD fragmentation is used for identification and compositional characterization of oligosaccharides attached to ECM glycoproteins. Figure 4A displays a representative spectrum obtained for a peptide labeled with ${ }^{18} \mathrm{O}$ after deglycosylation (indirect glycopeptide analysis). Figure 4B is a representative example of a spectrum obtained after analysis of intact glycopeptides from ECM extracts (direct glycopeptide analysis).

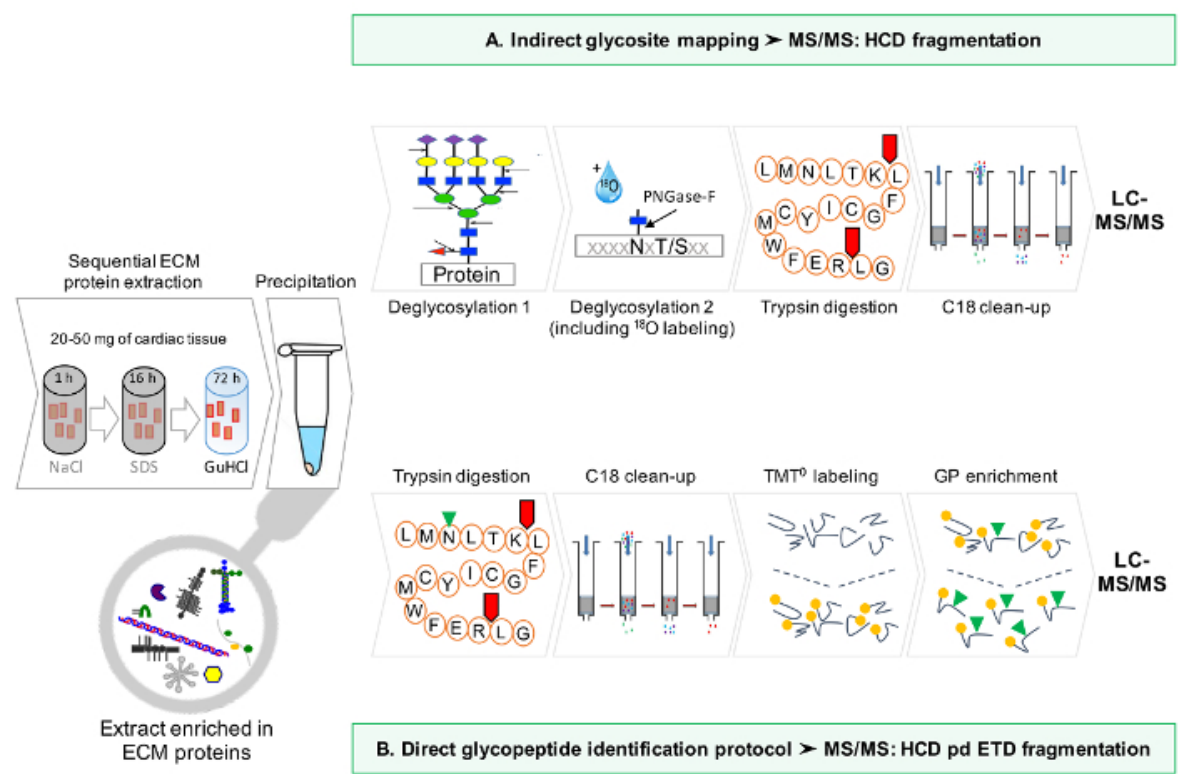

Figure 1: Method Overview. (A) After sequential enrichment for ECM proteins, LC-MS/MS analyses are performed on the deglycosylated extracts. (B) Alternatively, non deglycosylated ECM extracts are further enriched for glycopeptides. Please click here to view a larger version of this figure. 
A

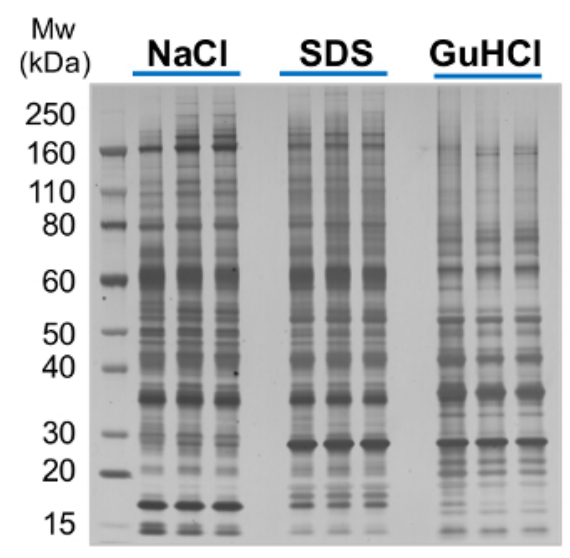

B

Decorin
(glycosylated)

Decorin

(GAG-deglycosylated)

Biglycan

Mimecan

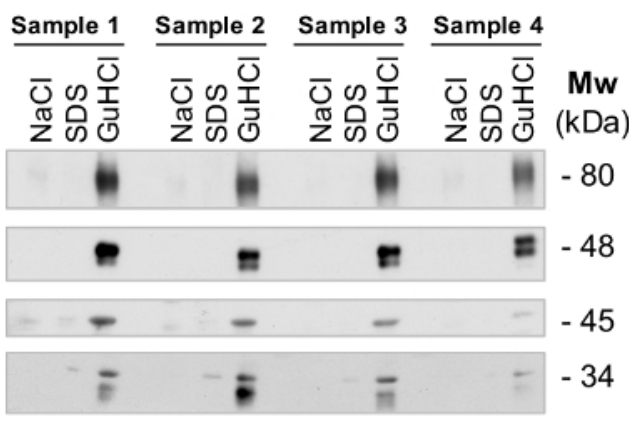

Figure 2: Extraction of ECM Proteins. (A) The 3 different extracts from the sequential extraction procedure ("English Quickstep") are complementary in their protein content. While SDS extracts are enriched in intracellular proteins, GuHCl extracts contain the majority of ECM proteins. Successful fractionation is visualized by the different silver staining pattern. (B) ECM proteins such as the small leucine-rich proteoglycans decorin, biglycan and mimecan are predominantly detected in the $\mathrm{GuHCl}$ extracts, with little presence in the SDS and $\mathrm{NaCl}$ extracts. Please click here to view a larger version of this figure. 
A

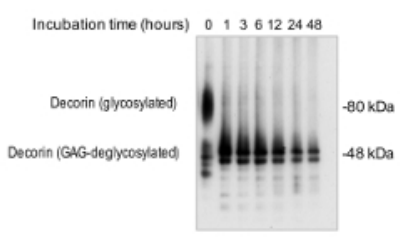

B

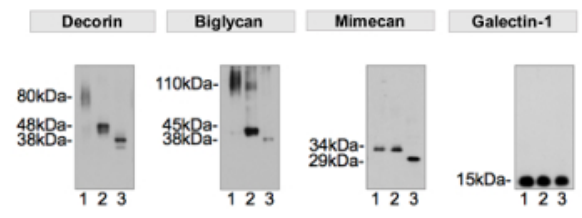

C

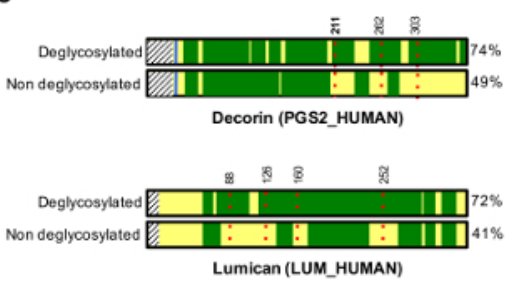

Figure 3. Analysis of Glycosylation. (A) Appropriate incubation times are required for complete deglycosylation. The example shows the effect of incubation time during removal of glycosaminoglycan chains from the glycoprotein decorin. (B) ECM glycoproteins are decorated with large and repetitive glycosaminoglycan chains and short and diverse $\mathrm{N}$ - and O-linked oligosaccharides. Lane 1 on each of the immunoblots represents untreated cardiac extracts. Lane 2 contains extracts treated with enzymes that digest glycosaminoglycans. Samples in lane 3 contain, in addition, enzymes for the removal of $\mathrm{N}$ - and O-linked oligossacharides. Galectin-1 is not glycosylated, hence there is no shift in protein size. Adapted from Lynch M, et al. ${ }^{4}$ (C) In LC-MS/MS analysis, samples treated with PNGase-F in the presence of $\mathrm{H}_{2}{ }^{18} \mathrm{O}$ achieve better sequence coverage (\%, on the right side) compared to non-deglycosylated samples. Dark green areas represent sequence coverage by LC-MS/MS. The red, dotted lines represent glycosites, with numbers indicating their amino acid position. Detection of glycosylation of decorin at position Asn ${ }^{211}$ $(\mathrm{N}$, highlighted in bold) is shown in detail as an example in Figure 4. Please click here to view a larger version of this figure.

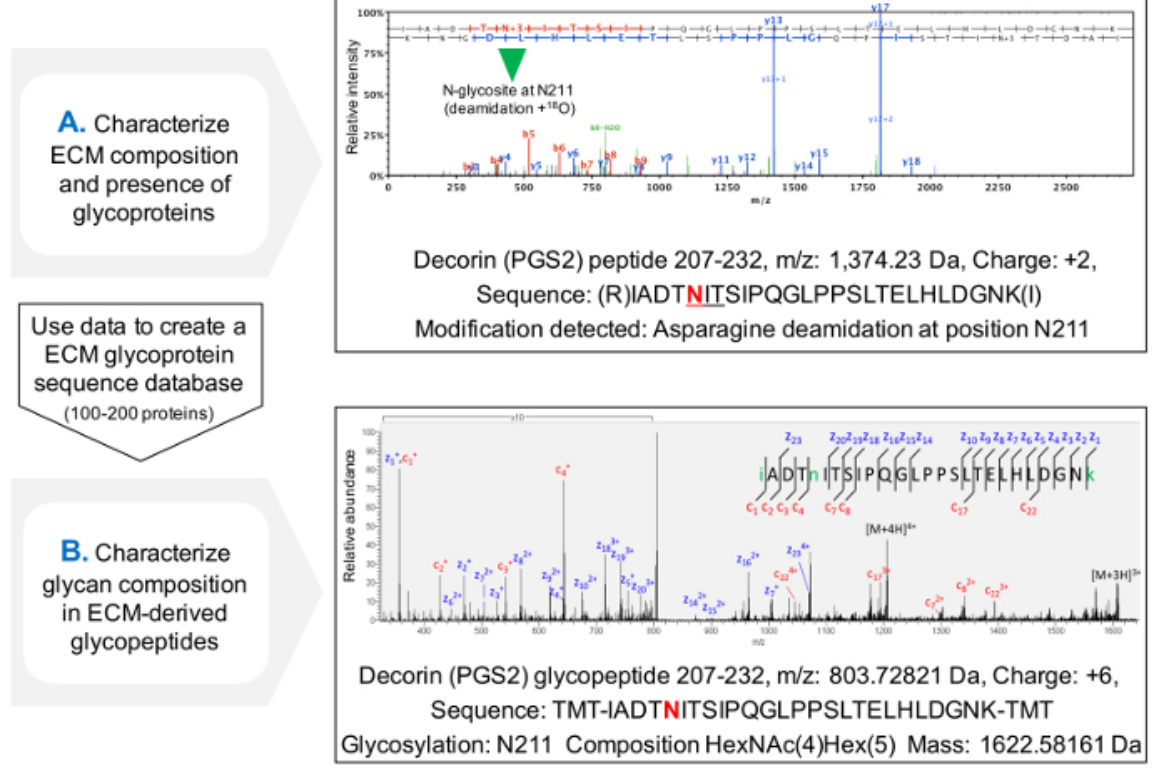

Figure 4. Glycopeptide Analysis by MS. (A) Using a shotgun proteomics approach on ECM enriched extracts, glycopeptides can be identified by the presence of deamidated asparagines within NxT/S sequons and labeled with ${ }^{18} \mathrm{O}$. The example shows a HCD MS/MS spectrum for a peptide of decorin containing the previously glycosylated Asn ${ }^{211}$. The data obtained can be used to create a customized database of ECM glycoproteins. (B) HCD-ETD fragmentation is used to analyze the glycopeptide enriched ECM extract. The ETD MS/MS spectrum allows the characterization of glycan composition. Please click here to view a larger version of this figure. 


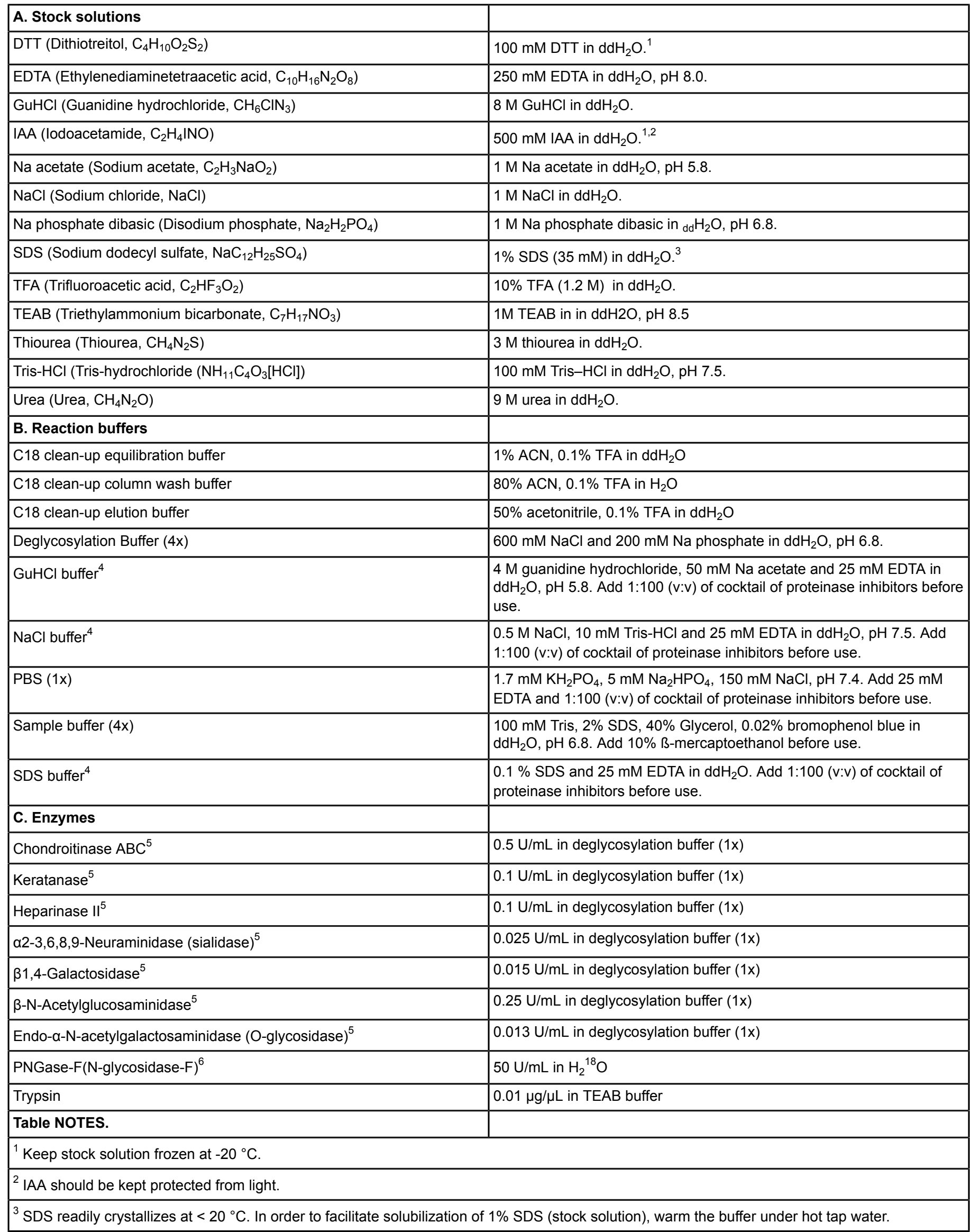




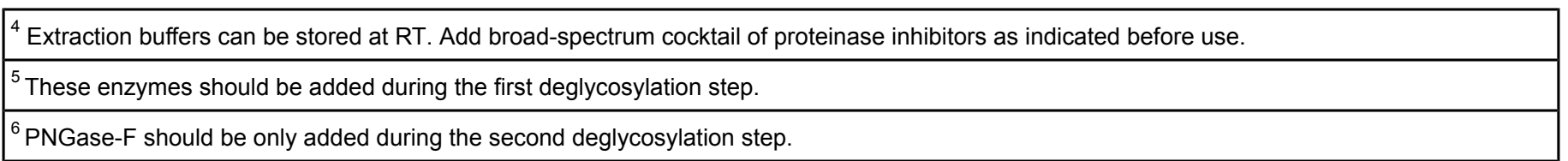

Table 1: Stock Solutions, Reaction Buffers and Enzymes. This table lists the composition of each stock solution and reaction buffer required for the extraction and subsequent processing (including enzymatic digestions) of cardiac ECM proteins prior to MS analysis.

\section{Discussion}

This proteomics protocol has been optimized over the last few years in our laboratory. Here, we used cardiac tissue, but only minor adjustments may be required for its application to other tissues. For example, the extraction protocol needs to take the cellularity of the tissue into consideration. Cardiac tissue is highly cellular compared to vascular tissue. When using vascular tissue, the SDS concentration can be lower (i.e. $0.08 \%$ ) and the decellularization time is shorter (i.e. $4 \mathrm{~h})^{11,12,13}$. The use of deglycosylation enzymes is crucial for LC-MS/MS analysis of ECM composition. However, incubation times need to be adjusted for different tissue types. For example, heparinase II required extended incubation times at $25^{\circ} \mathrm{C}$ when using samples such as skin, which are rich in basement membrane proteins (e.g. agrin, perlecan) (data not shown). Direct glycopeptide analysis can be performed on conditioned media from cells in culture ${ }^{15}$. Enrichment steps may not be required for the analysis of this simplified subproteome. Similar to $\mathrm{GuHCl}$ extracts, $\mathrm{NaCl}$ extracts are also amenable for glycoproteomics analysis with minor modifications. Other extraction protocols for enrichment of ECM proteins can be adapted to characterize ECM glycopeptides ${ }^{19,20}$.

Glycosylation is the most complex PTM ${ }^{5}$. Indirect identification of glycopeptides is achieved by the detection of deamidated Asn with incorporated ${ }^{18} \mathrm{O}$ at a NxT/S sequon. Deamidated Asn at other positions may represent false positives. Likewise, N-glycosylation must be considered in the context of protein ontologies: intracellular proteins containing a NxT/S sequon will not be glycosylated but might give rise to false positives. As current search algorithms do not allow for the screening of PTMs at pre-determined sequences only (i.e. Asn at NxT/ $\mathrm{S}$ ), manual filtering of the data is required. Identification of presence/absence of glycosylation at these positions can be compared between disease and control samples. There is no enzyme equivalent to PNGase F for O-deglycosylation (i.e. introducing a mass shift on threonine or serine). Therefore, the identification of O-glycosylation is restricted to direct glycopeptide analysis. Direct glycopeptide analysis is used to obtain compositional information of sugars attached to proteins, but does not provide structural information of the glycan. Moreover, the glycan composition is the result of glycan synthesis and processing after secretion.

Our 3-step extraction method for ECM proteins ("English Quickstep") ${ }^{6}$ has allowed characterizing the ECM in a variety of cardiovascular tissues. Fractionation of the tissue into several extracts is required to obtain a simplified ECM proteome as discussed elsewhere ${ }^{6}$. Intracellular proteins would otherwise contribute to an excessive dynamic range of protein abundances within the extracts that would hinder identification of less abundant ECM proteins. Moreover, intracellular proteins carry O-glycosylations ${ }^{5}$ that would complicate ECM glycopeptide enrichment and subsequent MS analysis. Other authors applied similar extraction methodologies to characterize for example lung ${ }^{21}$ and cartilage tissues ${ }^{10}$, however they did not pursue the analysis of glycosylation. Previous analysis of glycosylation focused on identification of glycosites only, require removal of the glycan from the protein core, and cannot assess O-glycosylation ${ }^{22,23}$. Lectin arrays and chemical enrichment are available for assessment of glycan types on biological samples based on their binding specificity, but these techniques cannot assign glycan types to specific proteins ${ }^{24}$ nor can they assess glycosylation sites.

Initially, we used gel electrophoresis prior to LC-MS/MS of ECM proteins. Although gel separation is useful in obtaining simplified protein fractions amenable to LC-MS/MS analysis, the latest instruments offer faster scan speeds. Thus, the electrophoretic separation step can be omitted. This provides an additional advantage as large ECM proteins, which are retained on top of the gel, are analyzed more efficiently. However, information regarding the $\mathrm{Mw}$ of the intact proteins is lost. The evaporation step prior to PNGase $\mathrm{F}$ deglycosylation ensures complete removal of regular $\mathrm{H}_{2} \mathrm{O}$ to minimize false negatives. Sugar residues (i.e. variable glycan masses) interfere with the separation by LC and compromise subsequent peptide identification by MS/MS. A pan-deglycosylation protocol is also recommended for proteomics analysis of ECM proteins not focused on glycosylation.

Proteomics can provide unprecedented insights into the ECM. Beyond structural support, glycans attached to the ECM are essential for hostpathogen interaction, cell-cell communication and the immune response ${ }^{25}$, i.e. allograft rejection after organ transplantation. Glycoproteomics will be an essential tool in glycobiology.

\section{Disclosures}

None.

\section{Acknowledgements}

JBB is a Career Establishment Fellow in the King's British Heart Foundation Centre. MM is a Senior Fellow of the British Heart Foundation (FS/13/2/29892). The study was supported by an excellence initiative (Competence Centers for Excellent Technologies - COMET) of the Austrian Research Promotion Agency FFG: "Research Center of Excellence in Vascular Ageing - Tyrol, VASCage" (K-Project number 843536) and the NIHR Biomedical Research Center based at Guy's and St. Thomas' National Health Service Foundation Trust and King's College London in partnership with King's College Hospital. 


\section{References}

1. Porter, K.E., \& Turner, N.A., Cardiac fibroblasts: at the heart of myocardial remodeling. Pharmacol. Ther. 123 (2), $255-278$ (2009).

2. Barallobre-Barreiro, J., et al. Proteomics analysis of cardiac extracellular matrix remodeling in a porcine model of ischemia/reperfusion injury. Circulation. 125 (6), 789-802 (2012).

3. Barallobre-Barreiro, J., et al. Glycoproteomics reveals decorin peptides with anti-myostatin activity in human atrial fibrillation. Circulation. 134 (11), 817-832 (2016).

4. Lynch, M., Barallobre-Barreiro, J., Jahangiri, M., \& Mayr, M. Vascular proteomics in metabolic and cardiovascular diseases. J. Intern. Med. 280 (4), 325-338 (2016).

5. Varki, A., \& Lowe, J.B. Biological Roles of Glycans. In: Varki A., et al, editors. Essentials of glycobiology. 2nd ed. Cold Spring Harbor NY: Cold Spring Harbor Laboratory Press (2009).

6. Barallobre-Barreiro, J., Lynch, M., Yin, X., \& Mayr, M. Systems biology - opportunities and challenges: The application of proteomics to study the cardiovascular extracellular matrix. Cardiovasc. Res. Sep 15 (2016).

7. Agnetti, G., Husberg, C., \& Van Eyk, J.E. Divide and conquer: the application of organelle proteomics to heart failure. Circ. Res. 108 (4), 512-526. (2011).

8. Mason, R.M., \& Mayes, R.W. Extraction of cartilage protein-polysaccharides with inorganic salt solutions. Biochem. J. 131 (13), 535-540 (1973).

9. Vogel, K.G., \& Peters, J.A. Isolation of proteoglycans from tendon. Methods. Mol. Biol. 171, 9-17 (2001).

10. Wilson, R., et al. Comprehensive profiling of cartilage extracellular matrix formation and maturation using sequential extraction and label-free quantitative proteomics. Mol. Cell. Proteomics. 9 (6), 1296-1313 (2010).

11. Barallobre-Barreiro, J. et al. Extracellular matrix remodeling in response to venous hypertension: proteomics of human varicose veins. Cardiovasc. Res. 110 (3), 419-430 (2016).

12. Didangelos, A., Yin, X., Mandal, K., Baumert, M., Jahangiri, M., \& Mayr, M. Proteomics characterization of extracellular space components in the human aorta. Mol. Cell. Proteomics. 9 (9), 2048-2062 (2010).

13. Didangelos, A., et al.Extracellular matrix composition and remodeling in human abdominal aortic aneurysms: a proteomics approach. Mol. Cell. Proteomics. 10 (8), M111.008128 (2011).

14. Grandoch, M., et al. Loss of biglycan enhances thrombin generation in apolipoprotein E-deficient mice: Implications for inflammation and atherosclerosis. Arterioscler Thromb. Vasc. Biol. 36 (5), e41-50 (2016).

15. Yin, X., Bern, M., Xing, Q., Ho, J., Viner, R., \& Mayr M. Glycoproteomic analysis of the secretome of human endothelial cells. Mol. Cell. Proteomics. 12 (4), 956-978 (2013).

16. Parker, B.L., et al. Quantitative N-linked glycoproteomics of myocardial ischemia and reperfusion injury reveals early remodeling in the extracellular environment. Mol. Cell. Proteomics. 10 (8), M110.006833 (2011).

17. Smith, P.K., et al. Measurement of protein using bicinchoninic acid. Anal. Biochem. 150 (1), $76-85$ (1985).

18. Pepinsky, R.B. Selective precipitation of proteins from guanidine hydrochloride-containing solutions with ethanol. Anal. Biochem. 195 (1), 177-181 (1991).

19. de Castro-Brás, L.E., et al. Texas 3-step decellularization protocol: looking at the cardiac extracellular matrix. J. Proteomics. 86, 43-52 (2013).

20. Naba, A., Clauser, K.R., \& Hynes, R.O. Enrichment of extracellular matrix proteins from tissues and digestion into peptides for mass spectrometry analysis. $J$ Vis Exp. (101), e53057 (2015).

21. Decaris, M.L., et al. Proteomic analysis of altered extracellular matrix turnover in bleomycin-induced pulmonary fibrosis. Mol. Cell. Proteomics. 13 (7), 1741-1752 (2014).

22. Zhang, H., Li, XJ., Martin, D.B., \& Aebersold, R. Identification and quantification of N-linked glycoproteins using hydrazide chemistry, stable isotope labeling and mass spectrometry. Nat. Biotechnol. 21 (6), 660-66 (2003).

23. Parker, B.L., et al. Site-specific glycan-peptide analysis for determination of N-glycoproteome heterogeneity. J. Proteome Res. 12 (12), 5791-5800 (2013).

24. Li, Y., et al. Simultaneous analysis of glycosylated and sialylated prostate-specific antigen revealing differential distribution of glycosylated prostate-specific antigen isoforms in prostate cancer tissues. Anal. Chem. 83 (1), 240-245 (2011).

25. Rienks, M., Papageorgiou, A.P., Frangogiannis, N.G., \& Heymans, S. Myocardial extracellular matrix: an ever-changing and diverse entity. Circ. Res. 114 (5), 872-888 (2014). 